\title{
Some Limit Theorems for Random Fields
}

\author{
Carla C. Neaderhouser $\star$ \\ Department of Mathematics, Texas A\&M University, College Station, Texas 77843, USA
}

\begin{abstract}
We prove a central limit theorem with remainder and an iterated logarithm law for collections of mixing random variables indexed by $Z^{d}, d \geqq 1$. These results are applicable to certain Gibbs random fields.
\end{abstract}

\section{Introduction}

In this paper we extend various classical limit theorems to collections of random variables indexed by $Z^{d}, d \geqq 1$. The study of such collections is motivated by Gibbs random fields. For example we may define our probability space $(\Omega, \mathscr{F}, P)$ in the following way. Let $\Omega=\{-1,+1\}^{Z^{d}}$, with $\mathscr{F}$ the $\sigma$-algebra generated by finite-dimensional cylinder sets. Then if $\Phi$ is a translation-invariant, finite-range, real-valued potential function on the finite subsets of $Z^{d}$ with $\Phi(\phi)=0$, the Gibbs state for the potential $\Phi$ is a probability measure $P$ on $(\Omega, \mathscr{F})$ for which, if $n \in Z^{d}$,

$$
P\left(\omega(n) \mid \mathscr{F}_{Z^{d}-\{n\}}\right)=\left(1+\exp \left(2 \sum_{A \in n}(\Phi(A)) \prod_{m \in A} \omega(m)\right)\right)^{-1}
$$

is a regular conditional probability distribution for the "spin" at site $n$ given the configuration on $Z^{d}-\{n\}$. Here $\mathscr{F}_{A}$ is the $\sigma$-algebra depending on the coordinates in the set $A \subset Z^{d}$.

In [2] a unique such $P$ is shown to exist for certain choices of $\Phi$. Moreover conditions on $\Phi$ which imply that mixing occurs are stated. That is, it is shown that for certain $\Phi$ and for $A, B \subset Z^{d}$ the dependence of sets in $\mathscr{F}_{A}$ on sets in $\mathscr{F}_{B}$ decreases as $d(A, B)$, the usual Euclidean distance between $A$ and $B$, increases, but may increase as $|A|$, the cardinality of $A$, increases.

In [5] the F. K. G. inequalities are used to determine conditions on $\Phi$ which imply that events at sites $n, m \in Z^{d}$ are positively correlated. For example if $\Phi$ is a nearest-neighbor potential

$$
\Phi(\{n, m\})>0 \text { for } d(n, m)=1
$$

is sufficient.

Thus we are led to study random variables $\left(X_{n}\right), n \in Z^{d}$, which are positively

* Partially supported by NSF Grant MSC 76-05828 and a grant from the Texas A\&M University College of Science 
correlated and which satisfy some sort of mixing condition. In the above example, for instance, we may consider $X_{n}(\omega)=\omega(n)$, the "spin" at $n$. We are interested here in asymptotic properties of sums $S_{A}=\sum_{n \in A} X_{n}$. In [9] it is shown that a sufficiently strong mixing condition together with positive correlation between sites and certain moment conditions implies a central limit theorem for the sequence $S_{A}$ when $A \uparrow Z^{d}$ sufficiently nicely. In this paper we show that the same properties of $P$ and the random variables $X_{n}$ may be used to estimate the speed at which $S_{A}$ converges to a normal random variable. This estimate is then used to obtain results about a.s. convergence, namely an iterated logarithm law and its consequences. The techniques used are modifications of those which work for $d=1$ [see e.g., 14, chapter 5.] The question of when the central limit theorem holds for random fields has, of course, been studied repeatedly (see, e.g., [3]), but questions about a.s. convergence seem not to have been looked at much up to now.

The statements of our results are in Section 3 and the proofs are in Section 5. Section 2 contains some necessary notation and some basic estimates. In Section 4 we discuss some of the known results on mixing for random variables indexed by $Z^{d}$. So far it seems that in models where mixing can be shown to occur it occurs at an exponential rate. However some of the results in [10] strongly suggest that slower mixing rates are possible, perhaps for infinite range potentials. Because of this and also because our proofs in the exponential case are easily modified to work for more slowly decreasing functions we have included such functions in the statements of the results.

\section{Notation and Some Basic Estimates}

Let $d \geqq 1$ be fixed. We assume throughout that we are given a probability space $(\Omega, \mathscr{F}, P)$, with $\Omega=\mathbb{R}^{Z^{d}}$ and $\mathscr{F}$ the $\sigma$-algebra generated by finite-dimensional cylinder sets, and a collection of real-valued random variables $\left(X_{n}\right), n \in Z^{d}$, with $X_{n} \in \mathscr{F}_{\{n\}}$. We also assume

$$
E\left(X_{n}\right)=0 \text { and } 0<E\left(X_{n}^{2}\right)<\sigma^{2} \text { for all } n \text {. }
$$

For simplicity we assume $A_{N}$ is a $d$-dimensional cube of side $N$ or sphere of radius $N$. We set

$$
\begin{aligned}
& S_{N}=\sum_{n \in A_{N}} X_{n}, \text { and } \\
& \Sigma_{N}^{2}=\operatorname{Var} S_{N}=E\left(\sum_{n \in A_{N}} X_{n}\right)^{2},
\end{aligned}
$$

and we assume that there is a $C>0$ for which, if $A \subset Z^{d}$,

$$
E\left(\sum_{n \in A} X_{n}\right)^{2} \geqq C|A| .
$$

Condition (2.2) is implied, of course, if the $X_{n}$ are positively correlated or independent and identically distributed or if events at sites $m$ and $n, m \neq n$, are sufficiently 
close to being uncorrelated. If the $X_{n}$ are independent we are reduced to the case $d=1$. Here we assume instead an asymptotic independence or "mixing".

Definition (2.3). We shall say the measure $P$ satisfies mixing condition $(M)$ if there exists a continuous function $\alpha:[1, \infty) \rightarrow(0, \infty)$ such that $\alpha(h) \downarrow 0$ as $h \uparrow \infty$ and, for $A, B \subset Z^{d}$ with $d(A, B)=h, E_{1} \in \mathscr{F}_{A}$ and $E_{2} \in \mathscr{F}_{B}$,

$$
\left|P\left(E_{1} E_{2}\right)-P\left(E_{1}\right) P\left(E_{2}\right)\right| \leqq \alpha(h)|A||B| .
$$

Obviously this definition is nontrivial only if $A$ and $B$ are both finite. Now $(M)$ implies the following estimates, the proofs of which may be found in [1] and [16]:

Lemma (2.4). Suppose $(M)$ holds, $A, B \subset Z^{d}$ with $d(A, B)=h$, and $f \in \mathscr{F}_{A}, g \in \mathscr{F}_{B}$ with $\|f\|_{p}<\infty$ and $\|g\|_{q}<\infty$ for some $p, q \geqq 1$, where the norms are with respect to the measure $P$. Then if $p, q, r \geqq 1$ satisfy $\frac{1}{p}+\frac{1}{q}+\frac{1}{r}=1$,

$$
|E(f g)-E(f) E(g)| \leqq(4 \alpha(h)|A||B|)^{1 / r}\|f\|_{p}\|g\|_{q} .
$$

If $p=q=\infty$

$$
|E(f g)-E(f) E(g)| \leqq 4 \alpha(h)|A||B|\|f\|_{\infty}\|g\|_{\infty} .
$$

\section{The Main Theorems}

Let $\phi(x)$ denote the distribution function of a normal random variable with mean 0 and variance 1 . Then we have the following results:

Theorem (3.1). Suppose (2.2) holds and $(M)$ holds with $\alpha(h)=e^{-\beta h}$ for some $\beta>0$. Suppose also that there is a $C>0$ with $E\left|X_{n}\right|^{5} \leqq C$ for all $n \in Z^{d}$. Then for $x \in \mathbb{R}$ and $d>1$

$$
P\left(X_{N} / \Sigma_{N}<x\right)=\phi(x)+O\left(\Sigma_{N}^{-(3 / 5 d)}\right) .
$$

If $d=1$

$$
P\left(X_{N} / \Sigma_{N}<x\right)=\phi(x)+O\left(\Sigma_{N}^{-1 / 2}\right) .
$$

Theorem (3.2). Suppose (2.2) holds and (M) holds with

$$
\int_{1}^{\infty} \alpha^{1 / 5}(h) h^{2 d-1} d h<\infty .
$$

Suppose also that there is a $C>0$ with $E\left|X_{n}\right|^{5} \leqq C$ for all $n \in Z^{d}$. Then for $x \in \mathbb{R}$

$$
P\left(X_{N} / \Sigma_{N}<x\right)=\phi(x)+O\left(\Sigma_{N}^{-(1 / 4 d)}\right) .
$$

Theorem (3.4). Suppose (2.2) holds and (M) holds with

$$
\alpha(h)=O\left(h^{-2 d-\varepsilon} \wedge h^{-3}\right)
$$

for some $\varepsilon>0$. Suppose there is a $C>0$ with $\left\|X_{n}\right\|_{\infty} \leqq C$ for all $n \in Z^{d}$. Then (3.3) is true.

These results slightly better the remainder terms obtained in [12] for $d=1$, 
but the error is still considerably larger than in the independent case, where the example of coin-tossing random variables, $X_{n}= \pm 1$, each with probability $1 / 2$, $d=1$, shows that $O\left(\Sigma_{N}^{-1}\right)$ is best possible.

These theorems have the following consequences:

Theorem (3.5). Suppose the $X_{n}$ satisfy the hypotheses of (3.1) or(3.2). If we set

$$
t_{N}=2 \Sigma_{N}^{2} \log \left(\log \Sigma_{N}^{2}\right)
$$

then

$$
\varlimsup_{N \rightarrow \infty} \frac{S_{N}}{t_{N}^{1 / 2}}=1 \text { a.s. }
$$

and

$$
\lim _{N \rightarrow \infty} \frac{S_{N}}{t_{N}^{1 / 2}}=-1 \text { a.s. }
$$

Theorem (3.9). Suppose the $X_{n}$ satisfy the hypotheses of $(3.4)$ with $\alpha(h)=$ $O\left(h^{-8 d-6-\varepsilon}\right)$ for some $\varepsilon>0$. Then (3.7) and (3.8) are true for $t_{N}$ given by (3.6).

Theorem (3.9), of course, improves (3.5) and (3.10) below only for $d \geqq 3$.

Corollary (3.10). If the $X_{n}$ satisfy the hypotheses of (3.5) or (3.8) and if $\left(a_{N}\right)_{N \geqq 1}$ are constants for which

$$
t_{N}^{1 / 2}=o\left(a_{N}\right)
$$

where $t_{N}$ is defined by (3.8) then

$$
\text { (3.11) } \lim _{N \rightarrow \infty} \frac{S_{N}}{a_{N}}=0 \text { a.s. }
$$

Notice that (3.10) implies

$$
\lim _{N \rightarrow \infty} \frac{S_{N}}{\sum_{N}^{p}}=0 \text { a.s. }
$$

for any $p>1$. In [9] it is shown that if $E\left(X_{n}^{4}\right) \leqq C$ for all $n$, and $(M)$ holds with

$$
\int_{1}^{\infty} \alpha^{1 / 2}(h) h^{d-1}<\infty,
$$

then (3.11) is true for $a_{N}=\Sigma_{N}^{2 k}, k>1 / 2$, if we replace "a.s." by "in probability", while (3.11) holds a.s. for $k \geqq 1$ if $d=1$ and $k>\frac{1}{2}\left(1+\frac{1}{d}\right)$ if $d>1$. These results do not require (2.2).

Results similar to those stated here are also proved in [13, especially Chapter 7] and [15], while in [17] a functional law of the iterated logarithm is obtained for $d \geqq 1$ for processes with independent increments. 


\section{Examples of Mixing}

In general determining when mixing occurs and at what rate seems to be an open problem. Perhaps the simplest method which has been developed to show that mixing occurs is that outlined in [2]. We describe the method and show how it applies to a simple example. Let us define $(\Omega, \mathscr{F})$ as in Section 1. For $\omega \in \Omega, n \in Z^{d}$, let ${ }_{n} \omega$ be $\left.\omega\right|_{Z^{d}-\{n\}}$ and let

$$
q\left({ }_{n} \omega, \cdot\right)=P\left(\left.\cdot\right|_{n} \omega\right)
$$

where $P(\cdot \mid \cdot)$ is given by $(1.1)$. For $\omega_{1}, \omega_{2} \in \Omega$ let $\left.\left.\rho\left(q_{n} \omega_{1}, \cdot\right), q_{n} \omega_{2}, \cdot\right)\right)$ be the distance in variation between the measures $q\left({ }_{n} \omega_{1}, \cdot\right)$ and $q\left({ }_{n} \omega_{2},{ }^{\circ}\right)$,

$$
\begin{aligned}
& \left.\left.\rho\left(q{ }_{n} \omega_{1}, \cdot\right), q{ }_{n} \omega_{2}, \cdot\right)\right) \\
& \left.\left.\left.\left.=\frac{1}{2}\left\{\mid q_{n} \omega_{1}, 1\right)-q_{n} \omega_{2}, 1\right)|+| q_{n} \omega_{1},-1\right)-q_{n} \omega_{2},-1\right) \mid\right\} .
\end{aligned}
$$

If $n, m \in Z^{d}, n \neq m$, let

$$
\left.\rho_{n, m}=\sup \rho\left(q{ }_{n} \omega_{1}, \cdot\right), q\left(\omega_{n}, \cdot\right)\right),
$$

where the sup is over all pairs ${ }_{n} \omega_{1},{ }_{n} \omega_{2}$ with ${ }_{n} \omega_{1}(l)={ }_{n} \omega_{2}(l)$ for $l \neq m$. Finally if $S \subset Z^{d}$ set

$$
\alpha\left(S,(S)_{h}\right)=\sum \rho_{n_{2}, n_{1}} \ldots \rho_{n_{k}, n_{k-1}},
$$

where the sum is over all paths $n_{1}, \ldots, n_{k}$ with $n_{1} \in S, n_{j} \neq S$ for $1<j \leqq k, n_{j+1} \neq n_{j}$ for $j=1, \ldots, k-1$, and $d\left(n_{k}, S\right) \geqq h$. Then the following is true:

Theorem (4.2). (Theorems 2 and 5, [2]). Suppose there is an $\alpha<1$ with

$$
\sum_{m \in Z^{d}-\{n\}} \rho_{n, m}<\alpha
$$

for all $n \in Z^{d}$. Then there is a unique (translation invariant) probability measure $P$ on $(\Omega, \mathscr{F})$ with regular conditional probability distribution given by (1.l) If in addition

$$
\sum_{n \in Z^{d}-\{m\}} \rho_{n, m}<\alpha
$$

for all $m \in Z^{d}$ and if $S_{1}, S_{2} \subset Z^{d}$ with $d\left(S_{1}, S_{2}\right) \geqq h$ then

$$
\sup _{A \in \mathscr{F}_{s_{1}}, B \in \mathscr{F}_{s_{2}}}|P(A B)-P(A) P(B)| \leqq \alpha\left(S_{1},\left(S_{1}\right)_{h}\right) .
$$

It is obvious that (4.5) is actually stronger than $(M)$, since the right hand side of (4.5) does not depend on $\left|S_{2}\right|$. Theorem (4.1) as stated in [2] is actually true with $\{-1,1\}$ replaced by any finite set and with the definitions above modified accordingly.

As a specific case of (4.1) let us consider the Ising model with

$$
\Phi(A)=\left\{\begin{array}{cl}
K / 2 & \text { if }|A|=1 \\
J / 2 & \text { if } A=\{n, m\} \\
0 & \text { otherwise }
\end{array} \text { and } d(n, m)=1\right\},
$$


where $K$ and $J$ are real numbers. Then if $x, y= \pm 1, d(n, m)=1$, and ${ }_{n} \omega(m)=y$,

$$
q{ }_{n}(\omega, x)=\left[1+\exp \left(K x+J x y+J x \sum \omega(j)\right)\right]^{-1},
$$

where the sum is over all $j \neq m$ with $d(j, n)=1$. Thus if we set $\mu=\min \left|K+J \sum \omega(y)\right|$ we find that

$$
\rho_{n, m}=\left\{\begin{array}{l}
\sinh |J| /(\cosh |J|+\cosh (\mu)) \text { if } d(n, m)=1 \\
0 \text { otherwise }
\end{array}\right\}
$$

and that $\delta_{n, m}$ does not depend on the sign of $K$ or of $J$. Since each $n \in Z^{d}$ has exactly $2 d$ neighbors at distance 1 , the sums in (4.3) and (4.4) are identical and thus Theorem (4.2) tells us that in this case there will be (a unique $P$ and) exponential mixing exactly when, for some $\alpha<1$,

$$
\sinh |J| /(\cosh |J|+\cosh \mu)<\alpha / 2 d .
$$

For example if $J=0$ then there is no interaction and trivially mixing occurs. If $K=0$ we must have tanh $|J|<1 / d$, and thus for $d=1$ there is mixing for all choices of $J$, while if $d=2$ we can show mixing only if $\tanh |J|<1 / 2$. When $K \neq 0$ and $J$ is fixed and nonzero, $K$ sufficiently large implies mixing while $|K|=$ $|J|$ implies mixing occurs only if $\sinh |J| /(1+\cosh |J|)<1 / 2 d$, a more stringent condition than in the case $K=0$. In fact it is known [7] that for this model, if $K \neq 0$, there is a unique $P$ with the given conditional distributions. However if $d=1$ and $K=J=2$ we have $\sinh |J| /(\cosh |J|+1)>3 / 4$ and (4.3) and (4.4) are not satisfied. Thus Theorem (4.2), while easy to apply, is not best possible.

A result similar to Theorem (4.2) is proved in [6, Theorem (4.24)]. In the example above, condition (a) Theorem (4.24) [6] is the same as our condition (4.4). The two conditions, in fact, would be equivalent for (1.1) with any pair potential and possible states \pm 1 . Mixing conditions of a slightly different type are obtained in [4].

Now if (4.3) is satisfied for this model the right-hand side of (4.5) is less than or equal to $\left|S_{1}\right| \alpha^{h} /(1-\alpha)$ and so $(M)$ certainly holds with $\alpha(h)=\alpha^{h} /(1-\alpha)$. Physically-motivated examples where mixing occurs at a slower than exponential rate seem hard to come by. Suppose, however, we set, for $n, m \in Z^{d},\|n, m\|=$ $\max _{1 \leqq i \leqq d}\left|n_{i}-m_{i}\right|$ and let $u(\{n, m\}, K)$ be the two point Ursell function for $\Phi(\{n\})=$ $K$, i.e.,

$$
u(\{n, m\}, K)=E\left(X_{n} X_{m}\right)-E X_{n} E X_{m} .
$$

Then in $[10]$ it is shown that whenever

$$
\lim _{\|n, m\| \rightarrow \infty} \sup \ln |\phi(n, m)| f\|n, m\|=0
$$

there is a $K_{0}$ for which

$$
\lim _{\|n, m\|} \sup \ln |u\{x, y\}, K| /\|n, m\|=0
$$

for almost all $K$ with $K<K_{0}$. By Lemma (2.4) if mixing occurs the function 
$\alpha(h)$ must satisfy, for $d(n, m)=h$,

$$
\alpha(h) \geqq A|u(\{n, m\}, K)|
$$

for some constant $A$. Thus physically-motivated examples of slower rates of mixing may exist.

\section{Proofs of the Theorems}

We first decompose $S_{N}$ into "almost independent" summands and a "negligible" summand, a standard technique for dealing with mixing random variables.

Definition (5.1). We define a sequence of annuli

$$
A_{N, 1}, A_{N, 1}^{\prime}, A_{N, 2}, A_{N, 2}^{\prime}, \ldots, A_{N, l_{N}}, A_{N, l_{N}}^{\prime}, A_{N, l_{N}+1}^{\prime},
$$

all contained in $A_{N}$, and random variables

$$
\begin{aligned}
Y_{N, j} & =\sum_{n \in A_{N}, j} X_{n}, \\
Z_{N, j} & =\sum_{n \in A_{N}^{\prime}, j} X_{N}, \\
Y_{N} & =\sum_{j} Y_{N, j}, \text { and } \\
Z_{N} & =\sum_{j} Z_{N, j},
\end{aligned}
$$

where we choose $A_{N, j}$ to be the largest annulus outside $A_{N, j-1}^{\prime}$ for which

$$
E\left(Y_{n, j}^{2}\right) \leqq N^{\eta}
$$

and $A_{N, j}^{\prime}$ to be the annulus outside $A_{N, j}$ with radius $k_{N}$, where $\eta$ and $k_{N}$ will be defined in the following lemmas.

Lemma (5.2). Suppose the hypotheses of (3.1) are satisfied. Then if we set

and

$$
\eta=d-1+\varepsilon, 0<\varepsilon<1,
$$

$$
k_{N}=r \log N,(d+2-2 \varepsilon) / \beta<r,
$$

we have

$$
\begin{aligned}
& E\left(Y_{N, j}^{2}\right)=N^{\eta}(1+o(1)), j \leqq l_{N} \\
& E\left(Y_{N, l_{N}+1}^{2}\right) \leqq N^{\eta}(1+o(1)) \\
& \sum_{j \leqq l_{N}+1} E\left(Y_{N, j}^{2}\right)=l_{N} N^{\eta}\left(1+O\left(N^{-\eta / 2}\right)\right), \\
& l_{N}=\left\{\begin{array}{l}
\sum_{N}^{2} N^{-\eta}\left(1+O\left(N^{-\varepsilon} r \log N\right)\right) \text { if } d>1 \\
\sum_{N}^{2} N^{-\eta}\left(1+O\left(N^{-\eta / 2}\right)\right) \text { if } d=1
\end{array}\right\}
\end{aligned}
$$


and

$$
E\left(\sum_{j \leqq l_{N}} Z_{N, j}\right)^{2}=N^{d-\varepsilon}(r \log N)(1+O(1)) .
$$

Lemma (5.7). If the hypotheses of (3.2) or (3.4) are satisfied, and if we set

$$
\eta=d-\varepsilon, 0<\varepsilon<1 \text {, }
$$

and

$$
k_{N}=N^{\varepsilon_{1}}, \varepsilon_{1} \geqq \varepsilon, 1-\varepsilon_{1}>\varepsilon, 1>\varepsilon+\varepsilon_{1},
$$

then (5.3), (5.4) and (5.5) are satisfied, while

$$
l_{N}=\Sigma_{N}^{2} N^{-\eta}\left(1+O\left(N^{\varepsilon_{1}+\varepsilon-1}\right)\right),
$$

and

$$
E\left(\sum_{j \leqq l_{N}} Z_{N, j}\right)^{2}=O\left(N^{d-1+\varepsilon+\varepsilon_{1}}\right) .
$$

Proof of (5.2) and (5.7). The proof is similar to the proof of Lemma 5 in [11]. Note that (2.1), (2.2), (2.4), and (5.3) together imply that $\Sigma_{N}^{2} \sim N^{d}$ and that the number of terms in a $Y_{N, j}$ is about $N^{\eta}$, while the number of terms in a $Z_{N, j}$ is $O\left(k_{N} N^{d-1}\right)$.

Theorem (5.9). Suppose the hypotheses of (3.1), (3.2), or (3.4) are satisfied. Then the central limit theorem holds for $X_{N} / \Sigma_{N}$, i.e., the distribution function of $X_{N} / \Sigma_{N}$ converges to the distribution function of $\phi$.

Proof. As usual, we prove that $E\left(\exp \left(i t X_{N} / \Sigma_{N}\right)\right.$ converges to $e^{-t^{2} / 2}$ for $t \in \mathbb{R}$. In each case $N^{2 d-\eta} l_{N} \alpha\left(k_{N}\right) \rightarrow 0$, so that applying (2.6) $l_{N}$ times gives

$$
E\left(\exp \left(i t Y_{N} / \Sigma_{N}\right)\right)=\prod_{j \leqq l_{N}+1} E\left(\exp \left(i t Y_{N, j} / \Sigma_{N}\right)\right)+o(1)
$$

Also (5.6) or (5.8) implies $E\left(\exp \left(i t X_{N} / \Sigma_{N}\right)\right)=E\left(\exp \left(i t Y_{N} / \Sigma_{N}\right)\right)+o(1)$, so it is enough to show that

$$
\prod_{j \leqq l_{N}+1} E\left(\exp \left(i t Y_{N, j} / \Sigma_{N}\right)\right)
$$

converges to $e^{-t^{2} / 2}$. Now if $p_{j}$ is the number of summands in $Y_{N, j},(2.5)$, the hypotheses of (3.1), (3.2), or (3.4), and the fact that $\Sigma_{n}^{2} \sim N^{d}$ can be used to show

$$
E\left(Y_{N, j}^{4}\right)=O\left(p_{j}^{2}\right)=O\left(N^{2 \eta}\right) .
$$

Thus

$$
\begin{aligned}
E\left|Y_{N, j}\right|^{3} & =O\left(N^{2 \eta}\right)^{3 / 4} \\
& =O\left(N^{3 \eta / 2}\right)
\end{aligned}
$$

and thus

$$
\Sigma_{N}^{-3} \sum_{j \leqq l_{N}+1} E\left|Y_{N, j}\right|^{3}=O\left(N^{-3 d / 2} l_{N} N^{3 \eta / 2}\right)=o(1)
$$


which proves (5.9) by Liapounov's theorem [8, p. 275].

Proof of (3.1),(3.4), and (3.6). We follow the procedure outlined in [12]. Set $g_{N}^{3}=$ $8 \Sigma_{N}^{-3} \sum_{j \leqq l_{N}+1} E\left|Y_{N, j}\right|^{3}$. Now, following the proof of Lemma 3 in [12], we may show that for large $N$ and for $0 \leqq t \leqq 2 / g_{N}^{3}$

$$
\left|\prod_{j<l_{N}+1} E\left(\exp \left(i t Y_{N, j}\right) / \Sigma_{N}\right)-e^{-t^{2} / 2}\right| \leqq e^{-t^{2} / 6} 2 t^{2}\left(t g_{N}^{3}+R\right)
$$

where

$$
R=\left\{\begin{array}{ll}
O\left(N^{-\varepsilon} r \log N\right) & \text { for (3.1), d>1 } \\
O\left(N^{-\eta / 2}\right) & \text { for (3.1), d=1 } \\
O\left(N^{\varepsilon_{1}+\varepsilon-1}\right) & \text { for (3.2) or (3.4) }
\end{array}\right\}
$$

Now the same proof as that of Lemma 4 in [12] gives

$$
E\left(\exp \left(i t Y_{N} / \Sigma_{N}\right)\right)-\prod_{j \leqq l_{N}+1} E\left(\exp \left(i t Y_{N, j} / \Sigma_{N}\right)\right)=O\left(t^{2}\right)
$$

uniformly in $0 \leqq t \leqq 1$, and then the proof of (14) in [12] yields that for $T=$ $\min \left(2 g_{N}^{-3}, \Sigma_{N}^{2}\right)$

$$
\int_{0}^{T}\left|E\left(\exp \left(i t Y_{N} / \Sigma_{N}\right)\right)-e^{-t^{2} / 2}\right| t^{-1} d t=O\left(g_{N}^{3}+R\right) .
$$

Thus by the basic inequality [8, p. 285]

$$
P\left(Y_{N} / \Sigma_{N}<x\right)-\phi(x)=O\left(g_{N}^{3}+R\right) \text {. }
$$

Now for $\varepsilon_{N}>0$

$$
\begin{aligned}
P\left(X_{N} / \Sigma_{N}<x\right)= & P\left(\left(\left(Y_{N}+Z_{N}\right) / \Sigma_{N}<x\right) \cap\left(\frac{\left|Z_{N}\right|}{\Sigma_{N}} \geqq \varepsilon_{N}\right)\right) \\
& +P\left(\left(\left(Y_{N}+Z_{N}\right) / \Sigma_{N}<x\right) \cap\left(\frac{\left|Z_{N}\right|}{\Sigma_{N}}<\varepsilon_{N}\right)\right) \\
= & P\left(Y_{N} / \Sigma_{N}<x+\varepsilon_{N}\right)+O\left(\frac{E\left(Z_{N}^{2}\right)}{\Sigma_{N}^{2} \varepsilon_{N}^{2}}\right) \\
= & \phi(x)+O\left(g_{N}^{3}+R\right)+O\left(\frac{E\left(Z_{N}^{2}\right)}{\sum_{N}^{2} \varepsilon_{N}^{2}}\right)+O\left(\varepsilon_{N}\right)
\end{aligned}
$$

by (5.12) and Chebyshev's inequality. Now set $\varepsilon=.395, \varepsilon_{N}=N^{-.3}$ if the hypotheses of (3.1) are satisfied with $d>1, \varepsilon=.5, \varepsilon_{N}=N^{-.35}$ if the hypotheses of (3.1) are satisfied with $d=1$, and $\varepsilon=1 / 4, \varepsilon_{1}=3 / 8, \varepsilon_{N}=N^{-1 / 8}$ if the hypotheses of (3.2) or (3.4) are satisfied. Together with (5.4), (5.11), and (5.6) or (5.8) this allows us to show that (5.13) gives the desired estimate.

Proof of (3.5) and (3.9). Since (3.7) implies (3.8) when we replace $S_{N}$ by $-S_{N}$, it is enough to show (3.7) is true. This is most easily accomplished in the manner 
outlined in [14, pp. $320 \mathrm{ff}$.$] , where results from [15] are proved in detail. First$ for $|\delta|<1$ and $\xi>0$

(5.14) $\left(\log \Sigma_{N}^{2}\right)^{-(1+\delta)^{2}(1+\xi)}<P\left(S_{N}>(1+\delta)\left(t_{N}^{1 / 2}\right)\right)<\left(\log \Sigma_{N}^{2}\right)^{-(1+\delta)^{2}(1-\xi)}$

follows from (3.1), (3.2), or (3.4). We also need that for $x>0$ and $N \geqq 1$

$$
P\left(\max _{J \leqq N} S_{J}>x\right) \leqq 2 P\left(S_{N}>x-2 \Sigma_{N}\right)+C N^{-\xi}
$$

for $0<\xi<1 / 4$ and $C>0$. This we may prove as follows:

Setting $B_{J}=\left(S_{I} \leqq x, I<J, S_{J}>x\right), 1 \leqq J \leqq N$, we have

$$
P\left(\max _{J \leqq N} S_{J}>x\right)=\sum^{\prime} P\left(B_{J}\right)+C N^{-\xi},
$$

where $\sum^{\prime}$ is over all $J$ with $P\left(B_{J}\right)>C N^{-(1+\xi)}$. So it suffices to show

$$
\sum^{\prime} P\left(B_{J}\right) \leqq 2 P\left(S_{N}>x-2 \Sigma_{N}\right) .
$$

Now

$$
\begin{aligned}
\sum_{J=1}^{N} P\left(\left|S_{N}-S_{J}\right| \leqq 2 \Sigma_{N} \mid B_{J}\right) P\left(B_{J}\right) & =\sum_{J=1}^{N} P\left(\left(\left|S_{N}-S_{J}\right| \leqq 2 \Sigma_{N}\right) \cap B_{J}\right) \\
& \leqq P\left(S_{N}>x-2 \Sigma_{N}\right)
\end{aligned}
$$

and thus it suffices to show

$$
P\left(\left|S_{N}-S_{J}\right|>2 \Sigma_{N} \mid B_{J}\right) \leqq 1 / 2
$$

if $P\left(B_{J}\right)>C N^{-(1+\xi)}$. Now for $N$ large enough and for some $\gamma, 0<\gamma+\xi<1 / 2$, $\alpha\left(N^{\gamma}\right) \leqq 10^{-5}$. Fix $\tau, 0<\tau<1 / 2$, and $J \geqq 1$ with $P\left(B_{J}\right)>C N^{-(1+\xi)}$. If $N-J>N^{\gamma}$, then

$$
\begin{aligned}
P\left(\left|S_{N}-S_{J}\right|>2 \Sigma_{N} \mid B_{J}\right) \leqq & P\left(\left|\sum_{n \in A_{J+N} \gamma-A_{J}} X_{n}\right|>\tau \Sigma_{N} \mid B_{J}\right) \\
& +P\left(\left|\sum_{n \in A_{N}-A_{J+N} \gamma} X_{n}\right|>(2-\tau) \Sigma_{N} \mid B_{J}\right) \\
\leqq & \frac{E\left|\sum_{n \in A_{J+N}-A_{J}} X_{n}\right|^{3}}{P\left(B_{J}\right) \tau^{3} \Sigma_{N}^{3}} \\
& +C N^{1+\xi} N^{2 d} \alpha\left(N^{\gamma}\right)+P\left(\left|\sum_{n \in A_{N}-A_{J+N} \gamma} X_{n}\right| \frac{3 \Sigma_{N}}{2}\right),
\end{aligned}
$$

where we have used Chebyshev's inequality and $(M)$ to obtain the first and second terms respectively. But

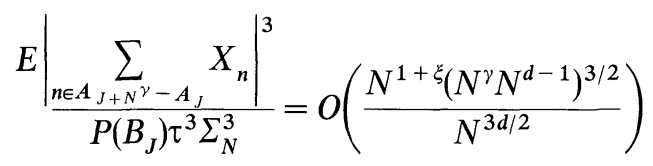




$$
\begin{aligned}
& =O\left(N^{\xi+\gamma-1 / 2}\right) \\
& <\frac{1}{100}
\end{aligned}
$$

for $N$ large enough, and, again by Chebyshev's inequality and $(M)$,

$$
\begin{aligned}
& C N^{1+\xi} N^{2 d} \alpha\left(N^{\gamma}\right)+P\left(\left|\sum_{n \in A_{N}-A_{J+N} \gamma} X_{n}\right|>\frac{3 \Sigma_{N}}{2}\right) \\
& =O\left(N^{2 d+1+\xi} \alpha\left(N^{\gamma}\right)+\frac{4}{9}\left(\frac{N^{d-1}\left(N-J-N^{\gamma}\right)}{N^{d}}\right)\right)=\frac{4}{9}+o(1),
\end{aligned}
$$

by the conditions on $\alpha(\cdot)$ specified by (3.5) or (3.9), if we choose $\xi=\frac{1}{100}, \gamma=\frac{4}{10}$. If, on the other hand, $N-J \leqq N^{\gamma}$,

$$
\begin{aligned}
P\left(\left|S_{N}-S_{J}\right|>2 \Sigma_{N} \mid B_{J}\right) & \leqq \frac{E\left|\sum_{n \in A_{N}-A_{J}} X_{n}\right|^{3} N^{1+\xi}}{8 \Sigma_{N}^{3} C} \\
& =O\left(\frac{N^{1+\xi}\left(N^{d-1+\gamma}\right)^{3 / 2}}{N^{3 d / 2}}\right) \\
& =O\left(N^{-(1 / 2)+\xi+\gamma}\right) .
\end{aligned}
$$

Thus (5.15) is true. Now, as in the standard proof of the law of the iterated logarithm, we have, for $\delta>0, \rho>1$, and $N_{j}=\rho^{j}, j \geqq 1$, by (5.15),

$$
\begin{aligned}
\sum_{j=1}^{\infty} P\left(\max _{N \leqq N_{J+1}} \frac{S_{N}}{t_{N}^{1 / 2}}>1+\delta\right) \leqq & \sum_{j=1}^{\infty} 2 P\left(S_{N_{j+1}}>(1+\delta) \varepsilon_{N_{J}}^{1 / 2}-2 \Sigma_{N_{J+1}}\right) \\
& +\sum_{j=1}^{\infty} C\left(N_{j+1}\right)^{-\xi} \\
& <\infty
\end{aligned}
$$

and thus

$$
P\left(\max _{N \leqq N_{J+1}} \frac{S_{N}}{t_{N}^{1 / 2}}>1+\delta \text { i.o. }\right)=0 .
$$

Now for $N>1$ and $\delta>0$, if we set

$$
C_{k}=\left(S_{N^{k}}-S_{N^{k-1}+N^{k / 2}}>(1-\delta)\left(2 \chi_{k}^{2} \log \left(\log \chi_{k}^{2}\right)\right)^{1 / 2}\right)
$$

where

$$
\chi_{k}^{2}=\operatorname{Var}\left(S_{N^{k}}-S_{N^{k-1}+N^{k / 2}}\right) \sim N^{k d}-\left(N^{k-1}+N^{k / 2}\right)^{d},
$$

then by (5.14), for $k$ large,

$$
P\left(C_{k}\right)>\left(\log \chi_{n}^{2}\right)^{-(1-\delta)^{2}(1+\xi)}
$$

for $|\delta|>1$ and $\xi>0$. If $\xi$ is chosen so that $(1-\delta)^{2}(1+\xi)<1$ then

$$
\sum_{k=1}^{\infty} P\left(C_{k}\right)=\infty
$$


since for $N$ and $k$ sufficiently large

$$
N^{k d}-\left(N^{k-1}+N^{k / 2}\right)^{d}>\frac{N^{k d}}{2} \text {. }
$$

Now, by $(M)$ and (3.5) or (3.9),

$$
\left|P\left(\bigcap_{k=n}^{n+m} C_{k}^{c}\right)-\prod_{k=n}^{n+m} P\left(C_{k}^{c}\right)\right|=O\left(\int_{N(k+1) / 2}^{\infty} \alpha(h) h^{4 d} d h\right)=o(1) .
$$

This estimate, together with (5.17) gives

$$
P\left(C_{k} \text { i.o. }\right)=1 \text {. }
$$

If

$$
B_{k}=\left(S_{N^{k+1}+N^{k} / 2}>-2\left(2 \tilde{\chi}_{k}^{2} \log \left(\log \tilde{\chi}_{k}^{2}\right)\right)^{1 / 2}\right),
$$

where

$$
\tilde{\chi}_{k}^{2}=\operatorname{Var}\left(S_{N^{k+1}+N^{k / 2}}\right) \sim\left(N^{k+1}+N^{k / 2}\right)^{d},
$$

then by (5.16) and (5.18)

$$
1=P\left(B_{k} \cap C_{k} \text { i.o. }\right)<P\left(S_{N^{k}}>\left(1-\delta^{\prime}\right) t_{N}^{1 / 2} \text { i.o. }\right)
$$

for $\delta^{\prime}>\delta$. Now (3.5) and (3.9) follow from (5.16) and (5.19).

\section{References}

1. Deo, C. M. : A note on empirical processes of strong-mixing sequences. Ann. Prob. 1, 870-875 (1973)

2. Dobrushin, R. L.: The description of a random field by means of conditional probabilities and conditions of its regularity. Theor. Prob. Appl. 13, 197-224 (1968)

3. Dobrushin, R. L., Tirozzi, B.: The central limit theorem and the problem of equivalence of ensembles. Commun. math. Phys. 54, 173-192 (1977)

4. Hegerfeldt, G. C., Nappi, C. R. : Mixing properties in lattice systems. Commun. math. Phys. 53, 1-7 (1977)

5. Helms, L. L. : Semigroups of operators and interacting particles. (unpublished manuscript)

6. Holley R. A., Stroock, D. W. : Applications of the stochastic Ising model to the Gibbs states. Commun. math. Phys. 48, 249-265 (1976)

7. Lebowitz, J. L., Martin-Löf, A. : On the uniqueness of the equilibrium state for Ising spin systems. Commun. math. Phys. 25, 276-282 (1972)

8. Loève, M. : Probability theory, 3rd ed., Princeton, New Jersey: Van Nostrand 1963

9. Neaderhouser, C. C. : Limit theorems for multiply-indexed mixing random variables, with applications to Gibbs random fields. Ann. Prob. 6, 207-215 (1978)

10. Penrose, O., Lebowitz, J. L. : On the exponential decay of correlation functions. Commun. math. Phys. 39, 165-184(1974)

11. Philipp, W. : The central limit problem for mixing sequences of random variables. Z. Wahrscheinlichkeitstheorie verw. Gebiete 12, 155-171 (1969)

12. Philipp, W.: The remainder in the central limit theorem for mixing stochastic processes. Ann. Math. Statist. 40, 601-609 (1969)

13. Philipp, W., Stout, W. F. : Almost sure invariance principles for partial sums of weakly dependent random variables. Am. Math. Soc. Mem. No. 161. Providence, Rhode Island: American Mathematical Society 1975 
14. Stout, W. F. : Almost sure convergence. New York: Academic Press 1974

15. Reznik, M. Kh. : The law of the iterated logarithm for some classes of stationary processes. Theor. Prob. Appl. 8, 606-621 (1968)

16. Volkonskii, V. A., Rozanov, Yu. A. : Some limit theorems for random functions I. Theor. Prob. Appl. 4, 178-197 (1959)

17. Wichura, M.: Some Strassen-type laws of the iterated logarithm for multiparameter stochastic processes with independent increments. Ann. Prob. 1, 272-296 (1973)

Communicated by J. L. Lebowitz

Received July 5, 1977; in revised form February 16, 1978 
